\title{
Tai Chi stabilisiert Parkinsonpatienten
}

\section{Durch ein Tai-Chi-Trainingsprogramm lassen sich bei Parkinsonpatienten mit leichter bis mittelschwerer Er- krankung, Gleichgewicht, Gangbild und Sturzneigung günstig beeinflus- sen.}

- Die Lebensqualität von Patienten mit Morbus Parkinson ist durch den zunehmenden Verlust der Fähigkeit zu stehen und zu gehen deutlich eingeschränkt. Mit Fortschreiten der Erkrankung kommt es zu Gangstörungen, die in Kombination mit dem Verlust der Stabilität im Stehen häufig zu Stürzen führen. Daher gehören Gleichgewichtsübungen und ein Muskeltraining zur obligaten physikalischen Übungsbehandlung bei Morbus Parkinson.

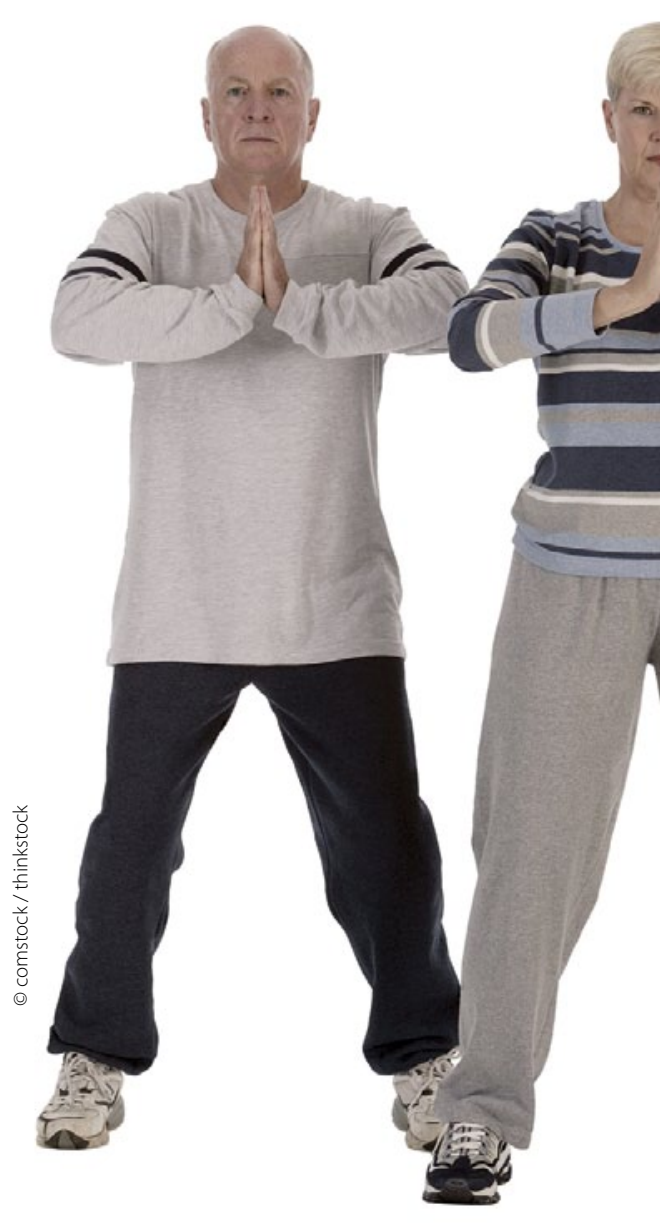

Die Übungstechnik Tai Chi spricht vor allem das Gleichgewicht an und ist in der Lage, bei älteren Personen Stürze zu verhindern. 195 Patienten mit Morbus Parkinson der Stadien 1-4 auf der Hoehn und Yahr-Skala wurden in eine von drei Übungsgruppen randomisiert. Gruppe 1 absolvierte Tai Chi-Übungen, Gruppe 2 ein spezielles Widerstandstraining und Gruppe 3 Dehnungsübungen. Die jeweils einstündigen Sitzungen fanden zweimal pro Woche über sechs Monate hinweg statt. Das primäre Studienziel war eine Änderung des Stabilitäts-Belastungstests im Vergleich zum Ausgangswert. Sekundäre Zielparameter beinhalteten die Schrittlänge, den Timed-up-and-go-Test, motorische Scores der Morbus-Parkinson-Bewertungsskala und die Anzahl von Stürzen.

Im Vergleich zum Ausgangswert ließ sich beim Stabilitäts-Belastungstest in der Tai-Chi-Gruppe eine Steigerung um 10\% beobachten. Auch beim Vergleich mit den beiden anderen Übungsgruppen (Widerstandsübungen und Stretching) beobachtete man eine Verbesserung von 5,6 bzw. 12\%. Die Schrittlänge hatte bei Patienten der Tai-ChiGruppe im Mittel um 10,3 cm zugenommen, die Gehgeschwindigkeit um $10,4 \mathrm{~cm} / \mathrm{s}$, die Kniestreckung um 13,9 Nm und die Kniebeugung um 5,1 Nm.
Im Verlauf der sechsmonatigen Studie ereigneten sich 381 Sturzereignisse bei 76 der 195 Teilnehmer. Die Inzidenzrate von Stürzen war in der Tai ChiGruppe um $67 \%$ niedriger als in der Stretching-Gruppe. Zwischen Tai Chi und den Widerstandsübungen bestand allerdings kein signifikanter Unterschied hinsichtlich der Sturzereignisse. Der Trainingseffekt der sechsmonatigen Tai-Chi-Übungen blieb über drei Monate nach der Intervention hinweg erhalten.

\section{Kommentar}

Ob man Tai Chi oder Widerstandsübungen der Muskulatur durchführt, ist für das funktionelle Ergebnis wahrscheinlich gar nicht entscheidend. Wichtig ist vor allem, dass Parkinsonpatienten, aber auch überhaupt alle älteren Menschen durch ein frühzeitig begonnenes und lebenslang fortgeführtes Muskeltraining versuchen, ihre Beweglichkeit, Balance und Muskelkraft möglichst lange zu erhalten. Wenn die Attraktivität derartiger Übungen durch den exotischen Namen Tai Chi gesteigert wird, kann das der Bewegung nur nützen. In unserer Gesellschaft hat diese Art der Sturzprophylaxe immer noch viel zu wenig Kultur. In chinesischen Städten gehört es zur selbstverständlichen Alltagskultur, dass in den frühen Morgenstunden in den Parks Tausenden von Menschen aller Altersgruppen Tai Chi-Übungen machen.

H. S. FÜESSL

\footnotetext{
- F. Li et al.

(Korres.: Dr. Li at the Oregon Research Institute, 1715 Franklin Blvd., Eugene, OR 97403; email fuzhongl@ori.org): Tai Chi and postural stability in patients with Parkinson's disease. New Engl. J. Med. 366 (2012) 511-519
}

Tai Chi: fernöstliche Sturzprophylaxe. 